\title{
Approximate Analysis of Power Offset over Spatially Correlated MIMO Channels
}

\author{
Guangwei YU, Xuzhen WANG \\ School of Information and Communication Engineering, Beijing University of Posts \\ and Telecommunications, Beijing, China \\ Email: \{yuguangwei1982,Gxitao\}@gmail.com
}

\begin{abstract}
Power offset is zero-order term in the capacity versus signal-to-noise ratio curve. In this paper, approximate analysis of power offset is presented to describe MIMO system with uniform linear antenna arrays of fixed length. It is assumed that the number of receive antenna is larger than that of transmit antenna. Spatially Correlated MIMO Channel is approximated by tri-diagonal toeplitz matrix. The determinant of tri-diagonal toeplitz matrix, which is fitted by elementary curve, is one of the key factors related to power offset. Based on the curve fitting, the determinant of tri-diagonal toeplitz matrix is mathematically tractable. Consequently, the expression of local extreme points can be derived to optimize power offset. The simulation results show that approximation above is accurate in local extreme points of power offset. The proposed expression of local extreme points is helpful to approach optimal power offset.
\end{abstract}

Keywords: MIMO, multiplexing gain, power offset, high snr region, toeplitz matrix

\section{Introduction}

Multiple-input multiple-output (MIMO) system is widely used in wireless communication to improve the performance. The spectral efficiency of MIMO channel is much higher than that over the conventional signal antenna channel. The research of MIMO includes two different perspectives: the first one concerns performance analysis in terms of error performance of practical systems, the second one concerns the study of channel capacity.

The design of communication schemes was mainly considered in the former perspectives with the aid of theoretical analysis and simulation [1][2], and [3]. For the latter, important parameters such as diversity gain [4], multiplexing gain [5][6] (referred to degree of freedom in other literatures), and power offset [7-9], and [10] were emphatically analyzed in the high signal-to-noise ratio (SNR) region. Furthermore, diversity and multiplexing tradeoff has been proposed in [11][12], and [13], that is to say diversity and multiplexing tradeoff can be obtained for a given multiple antenna channel. It is worth pointing out in [11] that the diversity and multiplexing tradeoff is essentially the tradeoff between the error probability and the data rate of a given system.

The multiplexing gain is not sufficient to accurately characterize the property of MIMO capacity. A more accurate representation of high SNR behavior in SNR-capacity curve is provided by an affine approximation to capacity, which includes both the multiplexing gain (i.e. slope) and power offset (i.e. zero-order term) [7]. High SNR power offset has been analyzed in [8] over multiple antenna Ricean channels. It was shown in [8] that the im- pact of the Ricean factor at high SNR region could be conveniently quantified through the corresponding power offset. In [9], high SNR power offset in multiple antenna communication was derived in detail. Achievable throughput was compared between the optimal strategy of dirty paper coding and suboptimal linear precoding techniques (zero-forcing and block diagonalization) in [10] on application of power offset. Hence, power offset is an important parameter in multiple antenna communication.

In many practical environments, signal correlation among the antenna array exists due to the scattering. Fading correlation and its effect on the capacity of multielement antenna system has been studied in [14]. Hence, analysis of spatially correlated MIMO channels has been another topic in the past few years, which necessitates the model of correlated channel. A general space-time correlation model for MIMO systems in mobile fading channel has been presented in [15]. The model in [15] was flexible and mathematically tractable. In [16], the correlated model of [15] has been used to investigate the capacity of spatially correlated MIMO Rayleigh-fading channels.

Recently, application of different antenna arrays in MIMO system has also been exploited. With the consideration of physical constraints imposed by maximum size of the antenna array, uniform linear array of fixed length has been used in [17] to analyze the asymptotic capacity of MIMO systems.

The uniform linear array of fixed length is also used in this paper to analyze the power offset of MIMO system over spatially correlated channel. On application of curve fitting, the determinant of tri-diagonal toeplitz matrix is mathematically tractable. Over spatially correlated chan- 
nel, the main result of this paper is the derived expression of local extreme points of power offset, using the uniform linear array of fixed length. To the best of our knowledge, the main result has not been presented in other literatures.

The rest parts are organized as follows. In Section 2, the basic definitions of multiplexing gain and power offset are presented. System model and correlation model are given in Section 3. Approximate analysis of power offset by fitting determinant curve of tri-diagonal toeplitz matrix is put forward in Section 4. Section 5 shows the simulation results. Finally, a brief conclusion is given in Section 6 .

Notation: In the following context, matrices and vectors are denoted by boldface upper case symbols and boldface lower case symbols, respectively. The transpose and Hermitian transpose are denoted by $(\cdot)^{T}$ and $(\cdot)^{H}$, respectively. The expected value is represented by $E[\cdot]$. $\|\cdot\|$ is used for the Euclidian norm.

\section{Basic Definition}

In the high SNR region, the capacity of single-user MIMO system of coherent reception is given by [18][19]

$$
C(S N R)=\min \left(n_{T}, n_{R}\right) \log _{2} S N R+o(1)
$$

where $n_{T}, n_{R}$ denote the number of transmit and receive antenna elements, respectively. $S N R$ is the signal to noise ratio. The MIMO capacity in high SNR region is a linear function of $\min \left(n_{T}, n_{R}\right)$, i.e. the stationary slope in SNR-capacity curve. In other words, any increase in $\max \left(n_{T}, n_{R}\right)$ is immaterial, i.e. without any impact to the slope. The slope is the so-called maximum multiplexing gain (degree of freedom).

If $n_{T}$ is fixed to a given value (generally speaking, $n_{T}$ is less than six, expressed as $\widehat{n_{T}}$ in the following context), and $n_{R} \geq n_{T}$, the multiplexing gain equals to a fixed value without consideration of correlation of channel response and the number of receive antenna elements. So power offset is introduced to compare the impact of channel property with the same value of multiplexing gain, and (1) is replaced by [9]

$$
\begin{aligned}
C(S N R) & =S_{\infty}\left(\log _{2}(S N R)-L_{\infty}\right)+o(1) \\
& =S_{\infty}\left(\frac{S N R_{d B}}{3 d B}-L_{\infty}\right)+o(1)
\end{aligned}
$$

where $S_{\infty}$ is multiplexing gain and $L_{\infty}$ is power offset in 3-dB units. When $S N R \rightarrow \infty$, the multiplexing gain and power offset can be computed by

$$
S_{\infty}=\lim _{S N R \rightarrow \infty} \frac{C(S N R)}{\log _{2}(S N R)}
$$

$$
L_{\infty}=\lim _{S N R \rightarrow \infty}\left(\log _{2}(S N R)-\frac{C(S N R)}{S_{\infty}}\right)
$$

In the high SNR region, SNR-capacity curve is approximately determined by multiplexing gain and power offset. Most channels, having the same multiplexing gain, may have very different capacities because of various values of the power offset. Hence, power offset is an important parameter to describe the capacity behavior in high SNR region.

\section{System Model and Correlation Mode}

The system model and channel correlation model are defined in this section. For MIMO system, the general baseband model is given by

$$
\mathbf{y}=\mathbf{H} \mathbf{x}+\mathbf{n}
$$

where $\mathbf{H}$ is the channel response matrix, and $\mathbf{x}=\left[\begin{array}{llll}x_{1} & x_{2} & \cdots & x_{\widehat{n_{T}}}\end{array}\right]^{T}$ is the input complex signal vector whose spatial covariance matrix normalized by the energy per dimension can be expressed as

$$
\boldsymbol{\Phi}=\frac{E\left(\mathbf{x x}^{H}\right)}{1 /{\widehat{n_{T}}} \times E\left[\|\mathbf{x}\|^{2}\right]}
$$

while $\mathbf{y}=\left[\begin{array}{llll}y_{1} & y_{2} & \cdots & y_{n_{R}}\end{array}\right]^{T}$ and $\mathbf{n}=\left[n_{1} n_{2} \cdots n_{n_{R}}\right]^{T}$ are the output vector and additive white Gaussian noise vector, respectively. Because of normalization and assumption of isotropic input, $\operatorname{tr}(\boldsymbol{\Phi})=\widehat{n_{T}}$ and $\boldsymbol{\Phi}=\mathbf{I}_{\widehat{n_{T}}}$, where $\mathbf{I}_{n}$ is $n \times n$ identity matrix.

Kronecker model [16] is used to describe the correlation of channel response. On the assumption of rich scattering environments and having no line of sight, the correlated channel response matrix is denoted by

$$
\boldsymbol{\Sigma}_{H}=\boldsymbol{\Sigma}_{T} \otimes \boldsymbol{\Sigma}_{R}
$$

where $\otimes$ is Kronecker product, $\boldsymbol{\Sigma}_{T}$ and $\boldsymbol{\Sigma}_{R}$ are correlated matrix of transmit and receive antenna, respectively. So the channel response matrix $\mathbf{H}_{\Sigma}$ is given by

$$
\mathbf{H}_{\Sigma}=\boldsymbol{\Sigma}_{R}^{1 / 2} \mathbf{H} \boldsymbol{\Sigma}_{T}^{1 / 2}
$$

where $\mathbf{H}$ is channel response matrix whose elements are independent and identical distribution random variables. Without loss of generality, we assume that the distance between transmit antenna elements is large enough to neglect the correlation at the transmit node. Consequently, without considering the antenna correlation at the transmit node, the correlation matrix $\boldsymbol{\Sigma}_{T}$ is identity matrix, and the receive antenna correlation model $\boldsymbol{\Sigma}_{R}$ is given by [15][16]. 


$$
\Sigma_{R}(i, j)=\frac{I_{0}\left(\sqrt{\eta^{2}-4 \pi^{2} d_{i j}^{2}+j 4 \pi \eta \sin (\mu) d_{i j}}\right)}{I_{0}(\eta)}
$$

where $\eta \in[0, \infty)$ is the so-called AOA(angle of arrival), $\mu \in[-\pi, \pi)$ is average value of AOA, $d_{i j}$ is normalized distance between antenna array elements, that is to say $d_{i j}=\widehat{d_{i j}} / \lambda, \widehat{d_{i j}}$ is the factual distance between $\mathrm{i}$ and $\mathrm{j}$ antenna array element, $\lambda$ is wave length, $I_{n}(\cdot)$ is n-order modified Bessel function. It is obvious that $\boldsymbol{\Sigma}_{R}(i, j)$ is real symmetric toeplitz matrix.

On the assumption of isotropic scattering, the correlation matrix at the receive node is simplified to

$$
\Sigma_{R}(i, j)=J_{0}\left(2 \pi d_{i j}\right)
$$

where $J_{n}(\cdot)$ is $\mathrm{n}$ order Bessel function, and $\boldsymbol{\Sigma}_{R}(i, j)$ can be simplified to tri-diagonal toeplitz matrix through the simulation results illustrated by Figure 1 and Figure 2.

$$
\boldsymbol{\Sigma}_{R}=\left[\begin{array}{ccccc}
1 & \alpha & & & \\
\alpha & \cdot & \cdot & & \\
& \cdot & \cdot & \cdot & \\
& & \cdot & \cdot & \alpha \\
& & & \alpha & 1
\end{array}\right]_{n_{R} \times n_{R}}
$$

$$
\alpha=J_{0}\left(2 \pi \frac{L_{R}}{n_{R}-1}\right), \quad 1 \leq L_{R} \leq 10
$$

where $L_{R}$ is the fixed length of receive antenna array normalized by $\lambda$. In this paper, $L_{R}$ is assumed to be in the range from 1 to 10 .

In the Figure 1 and 2, power offset approximation based on tri-diagonal, five-diagonal, and seven-diagonal toeplitz matrix is obtained versus the number of receive antenna with various value of $L_{R}$. Furthermore, power offset over independent channel is also provided as lower bound for numerical comparison. It can be seen from Figure 1 and 2 that tri-diagonal toeplitz matrix can be used to approximate the correlation model at the receive node.

\section{Approximate Analysis of Power Offset}

Depending on the discussion in Section 3, tri-diagonal toeplitz matrix can be used to approximate the correlation model at the receive node. In this section, the determinant curve of tri-diagonal toeplitz matrix is fitted by an elementary function, which simplifies the approximate analysis of power offset.

Expression of power offset for MIMO system over correlated channel is derived in [9], and the expression is given by

$$
\begin{aligned}
L_{\infty}= & \log _{2} \widehat{n_{T}}-\frac{1}{\widehat{n_{T}} \ln 2} E\left(\ln \operatorname{det}\left(\mathbf{W}^{H} \boldsymbol{\Lambda}_{R} \mathbf{W}\right)\right) \\
& -\frac{1}{\widehat{n_{T}}} \log _{2} \operatorname{det}\left(\boldsymbol{\Lambda}_{T} \mathbf{P}\right)
\end{aligned}
$$

where

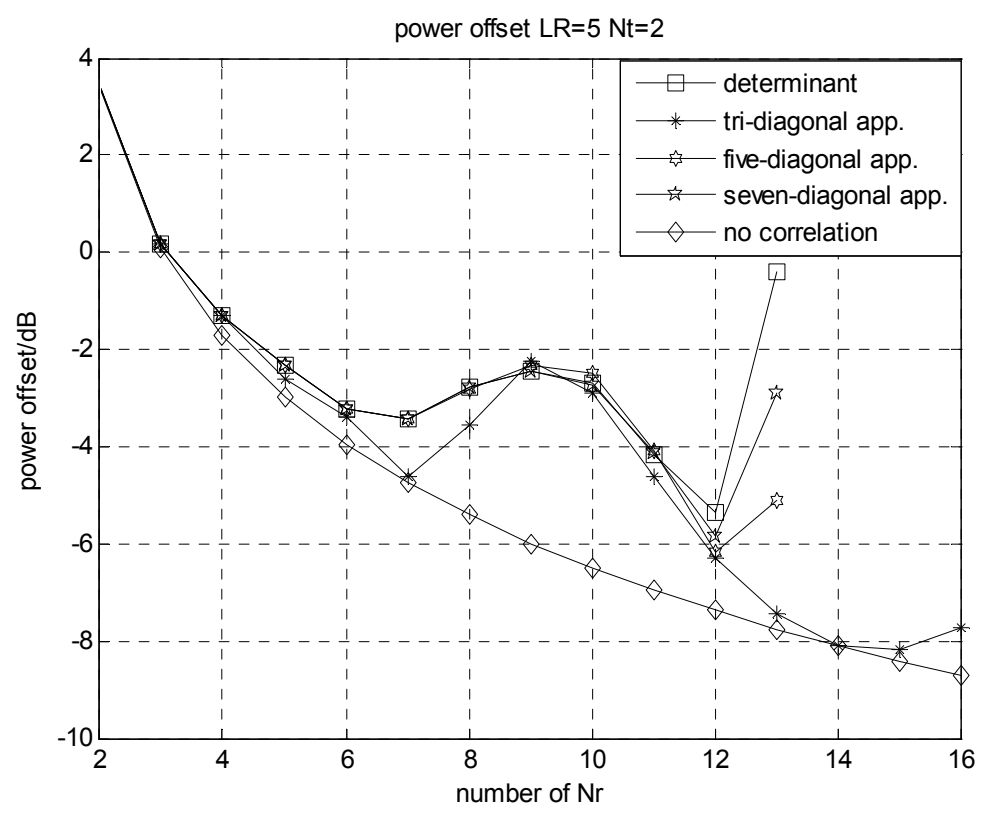

Figure 1. Approximate analysis of power offset based on 3, 5, and 7 diagonal toeplitz matrix with $L_{R}=5, \widehat{n_{T}}=2$ 


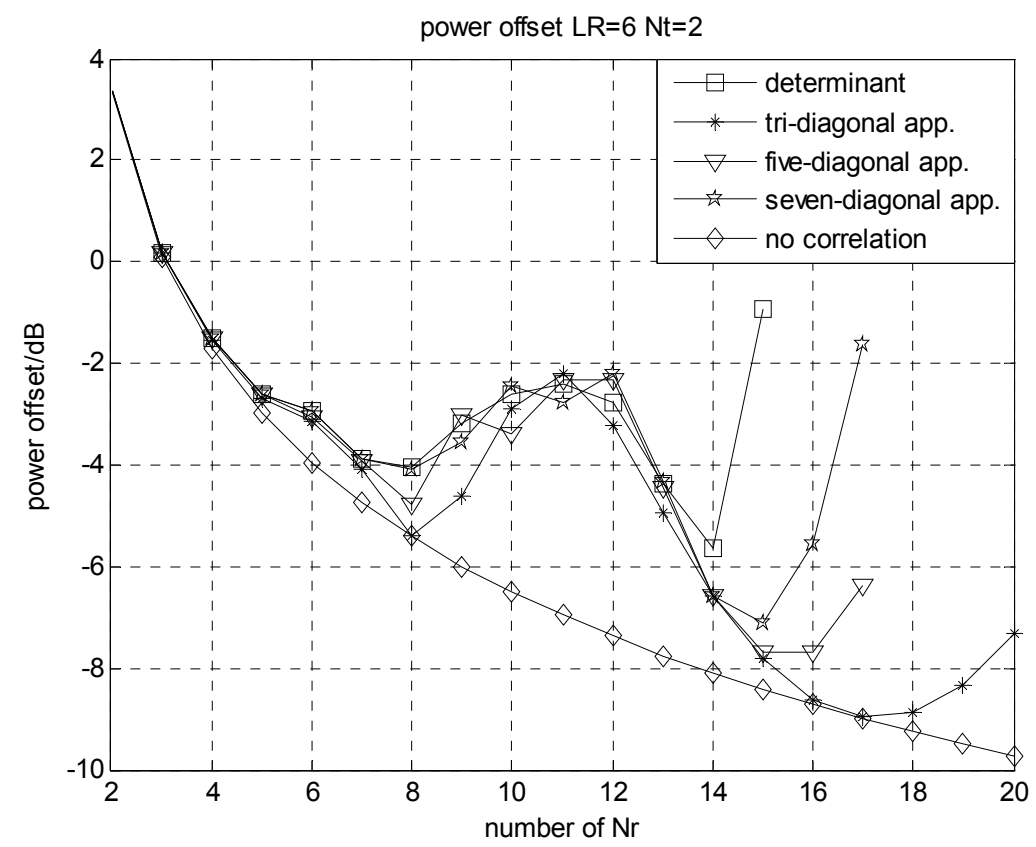

Figure 2. Approximate analysis of power offset based on 3, 5, and 7 diagonal toeplitz matrix with $L_{R}=6, \widehat{n_{T}}=2$

where $\boldsymbol{\Lambda}_{T}$ and $\boldsymbol{\Lambda}_{R}$ are diagonal matrix whose elements are eigenvalues of $\boldsymbol{\Sigma}_{T}$ and $\boldsymbol{\Sigma}_{R}$, respectively. $\mathbf{P}$ is also diagonal matrix whose elements are eigenvalues of normalized spatial covariance matrix $\boldsymbol{\Phi}$, so $\mathbf{P}$ is identity matrix and $1 / \widehat{n_{T}} \log _{2} \operatorname{det}\left(\boldsymbol{\Lambda}_{T} \mathbf{P}\right)$ is constant zero. $\boldsymbol{\Lambda}_{R}$ can be derived from (11). It is obvious that $\boldsymbol{\Lambda}_{R}$ is full rank matrix, and then (13) is simplified to

$$
L_{\infty}=\log _{2} \widehat{n_{T}}+\left(f\left(n_{R}\right)+g\left(n_{R}\right)\right)
$$

where $f\left(n_{R}\right)$ and $g\left(n_{R}\right)$ can be described by

$$
\begin{gathered}
f\left(n_{R}\right)=-\frac{E\left(\ln \operatorname{det}\left(\mathbf{W}^{H} \mathbf{W}\right)\right)}{\widehat{n_{T}} \ln 2} \\
g\left(n_{R}\right)=-\frac{\ln \operatorname{det}\left(\boldsymbol{\Lambda}_{R}\right)}{\widehat{n_{T}} \ln 2}
\end{gathered}
$$

Because $\mathbf{W}^{H} \mathbf{W}$ is wishart matrix and nonsingular with probability $1, f\left(n_{R}\right)$ is reduced to [9]

$$
\begin{aligned}
f\left(n_{R}\right) & =-\frac{1}{\widehat{n_{T}} \ln 2} E\left(\log _{e} \operatorname{det}\left(\mathbf{W}^{H} \mathbf{W}\right)\right) \\
& =-\frac{1}{\widehat{n_{T}} \ln 2} \sum_{l=0}^{\widehat{n_{T}}-1} \psi\left(n_{R}-l\right)
\end{aligned}
$$

where $\psi(\cdot)$ is digamma function and the definition of digamma function is

$$
\psi(n)=-\gamma+\sum_{l=1}^{n-1} \frac{1}{l}
$$

where $\gamma$ is Euler-Mascheroni constant, and

$$
\gamma=\lim _{n \rightarrow \infty}\left(\sum_{l=1}^{n} \frac{1}{l}-\log _{e} n\right) \approx 0.5772
$$

When it comes to function $g\left(n_{R}\right)$, the derivation of $g\left(n_{R}\right)$ can be expressed as

$$
g\left(n_{R}\right)=-\frac{1}{\widehat{n_{T}} \ln 2} \ln \operatorname{det}\left(\boldsymbol{\Lambda}_{R}\right)=-\frac{1}{\widehat{n_{T}} \ln 2} \ln \operatorname{det}\left(\boldsymbol{\Sigma}_{R}\right)
$$

It can be found from (20) that $g\left(n_{R}\right)$ is only determined by the determinant of $\boldsymbol{\Sigma}_{R}$, and the determinant of $n_{R}$ order tri-diagonal toeplitz matrix $\boldsymbol{\Sigma}_{R}$ can be computed by [20].

$$
\operatorname{det} \boldsymbol{\Sigma}_{R}=\left\{\begin{array}{l}
\frac{\left(1+\sqrt{1-4 \alpha^{2}}\right)^{n_{R}+1}-\left(1-\sqrt{1-4 \alpha^{2}}\right)^{n_{R}+1}}{2^{n_{R}+1} \sqrt{1-4 \alpha^{2}}} \\
\left(1-4 \alpha^{2} \neq 0\right) \\
\frac{n_{R}+1}{2^{n_{R}}} \\
\left(1-4 \alpha^{2}=0\right)
\end{array}\right.
$$

With the aid of numerical computation and mathematical analysis, the curve of the determinant of tri-diagonal toeplitz matrix can be fit by some tractable elementary 
function. When $1-4 \alpha^{2} \neq 0$ in $(21)$, $\operatorname{det} \Sigma_{R}$ can be approximated by the fitted curve as follow:

$$
h\left(n_{R}\right)=1-5 \alpha^{2}=1-5 J_{0}\left(2 \pi \frac{L_{R}}{n_{R}-1}\right)^{2}
$$

The approximation between the fitted curves and determinant curves of tri-diagonal toeplitz matrix are illustrated in Figure 3 and Figure 4. From Figure 3 and 4, we can find that the fitting curve can exactly approach the extreme points of the determinant of tri-diagonal toeplitz matrix. However, the expression is much simpler. In other words, the further derivation using the fitted curve is tractable using well-known mathematical software such as WOLFRAM MATHEMATICA.

In the following context, power offset is analyzed in detail. The approximate analysis of power offset is based on the piecewise function of $n_{R}$. The condition $1-5 \alpha^{2}>0$ in (22) is necessary due to $\operatorname{det} \boldsymbol{\Sigma}_{R}>0$, so the number of receiver antenna $n_{R}$ can not be large than $4 L_{R}+1$. Moreover, it shows from the conclusion in [21] that the effect of correlation can be neglected when the normalized length of receive antenna array $L_{R}$ is larger than one wave length, that is to say $n_{R}>L_{R}+1$. Hence, the considered range of the number of receive antenna is $L_{R}+1<n_{R}<4 L_{R}+1$.

With the discussion above, when $n_{R} \leq L_{R}+1$, the normalized length of receive antenna array $L_{R}$ is large than one wave length and the effect of correlation can be neglected, that is to say $g\left(n_{R}\right)=0$ and the power offset is expressed as

$$
L_{\infty}=\log _{2} \widehat{n_{T}}-\frac{1}{\widehat{n_{T}} \ln 2} \sum_{l=0}^{\widehat{n_{T}}-1} \psi\left(n_{R}-l\right)
$$

When it comes to the range of $L_{R}+1<n_{R}<4 L_{R}+1$, the normalized length of receive antenna array $L_{R}$ is smaller than one wave length and the effect of correlation can not be neglected. In other words, $g\left(n_{R}\right) \neq 0$. However, for a given value of $\widehat{n_{T}}$, the first-order derivative $\left(f\left(n_{R}\right)\right.$ is assumed to be continuous) of $f\left(n_{R}\right)$ is obtained by

$$
\begin{aligned}
\frac{d f\left(n_{R}\right)}{d n_{R}} & =\left(-\frac{1}{\widehat{n_{T}} \ln 2} \sum_{l=0}^{\widehat{n_{T}}-1} \psi\left(n_{R}-l\right)\right)^{\prime} \\
& =-\frac{1}{\widehat{n_{T}} \ln 2} \sum_{l=0}^{\widehat{n_{T}}-1} \psi_{1}\left(n_{R}-l\right) \approx 0
\end{aligned}
$$

where $\psi_{1}(\cdot)$ is trigamma function. Hence, it shown from (24) that $f\left(n_{R}\right)$ approximately equal to the constant $C$ (approximation accuracy is related to the value of $\widehat{n_{T}}$ ). The power offset is expressed as

$$
L_{\infty} \approx \log _{2} \widehat{n_{T}}+g\left(n_{R}\right)+C
$$

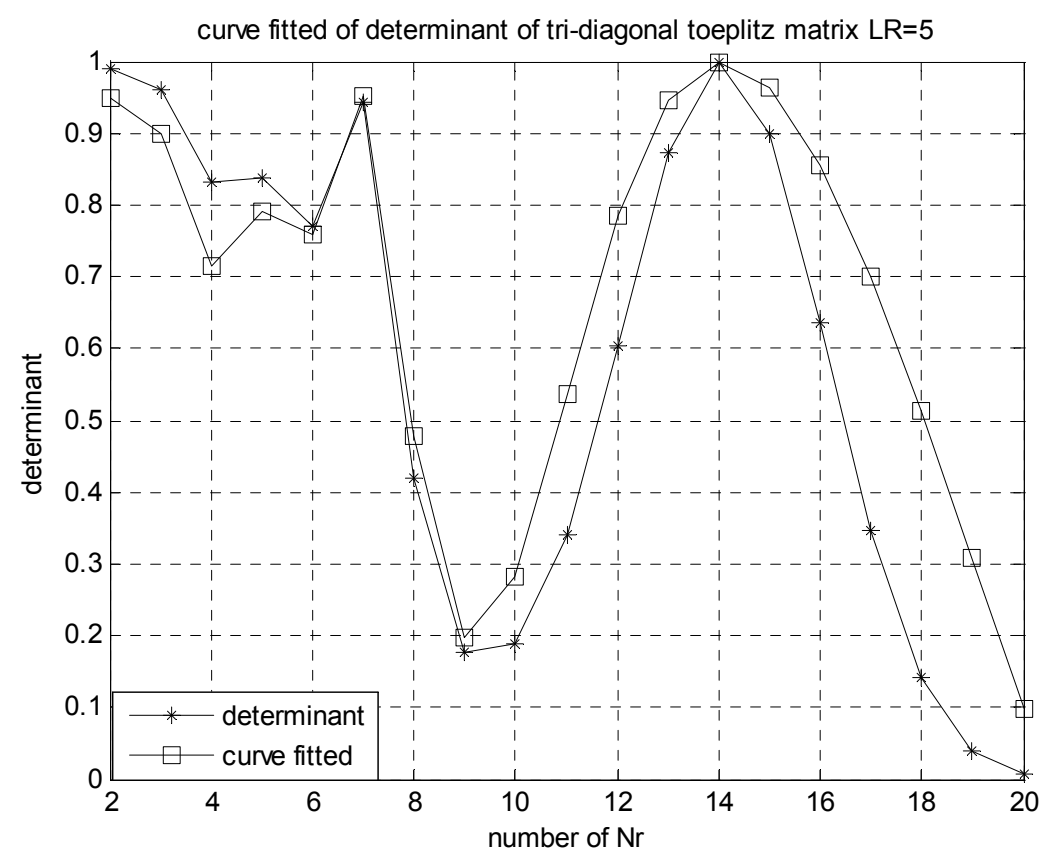

Figure 3. Approximation of determinant of tri-diagonal toeplitz matrix by curve fitting and $L_{R}=5$ 


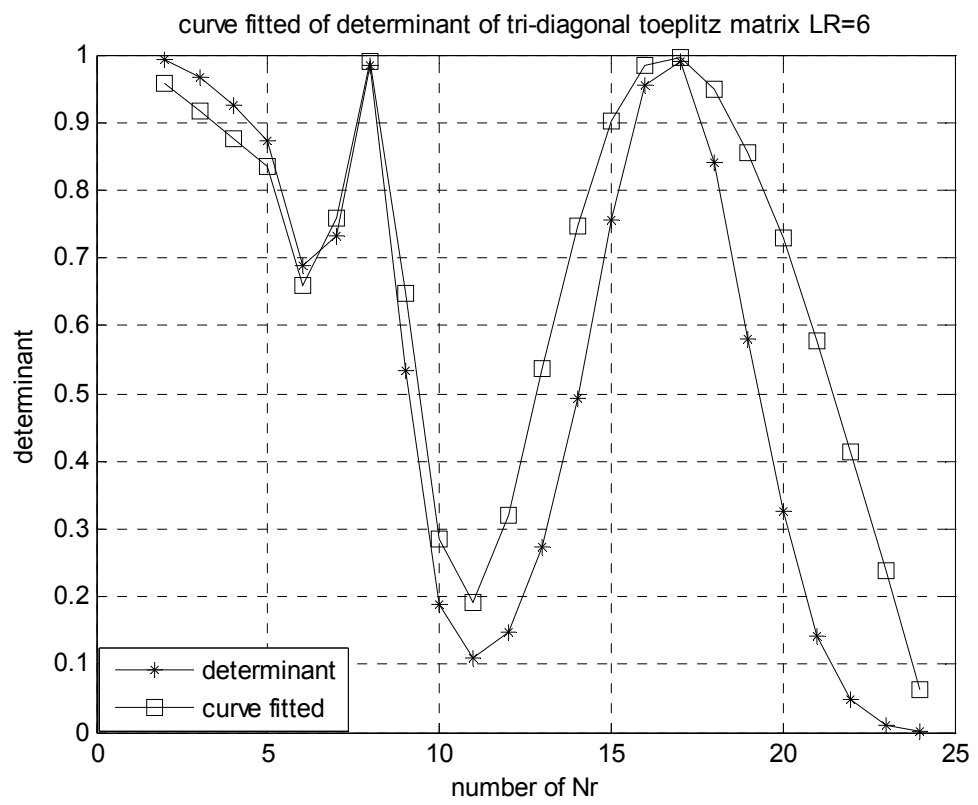

Figure 4. Approximation of determinant of tri-diagonal toeplitz matrix by curve fitting and $L_{R}=6$

Without loss of generality, the constant $C$ can be neglected in the approximate computation. Hence, the power offset in (25) is only determined by $g\left(n_{R}\right)$. From (22), the curve of the determinant of tri-diagonal toeplitz matrix can be fitted by $h\left(n_{R}\right)=1-5 \alpha^{2}$, where $\alpha=J_{0}\left(\frac{2 \pi L_{R}}{n_{R}-1}\right)$. The first order derivative $\left(h\left(n_{R}\right)\right.$ is assumed to be continuous) of $h\left(n_{R}\right)$ can be computed by

$$
\frac{d h\left(n_{R}\right)}{d n_{R}}=-\frac{4 \pi L_{R} J_{0}\left(\frac{2 \pi L_{R}}{n_{R}-1}\right) J_{1}\left(\frac{2 \pi L_{R}}{n_{R}-1}\right)}{\left(n_{R}-1\right)^{2}}
$$

where $L_{R}+1<n_{R}<4 L_{R}+1$, that is to say $\frac{\pi}{2}<\frac{2 \pi L_{R}}{n_{R}-1}<2 \pi$. In the range above, function $J_{0}\left(\frac{2 \pi L_{R}}{n_{R}-1}\right)$ in (26) has two zero points, i.e. $P_{1}=\frac{2 \pi L_{R}}{n_{R}-1}=2.4048 \quad$ and $\quad P_{2}=\frac{2 \pi L_{R}}{n_{R}-1}=5.5201, \quad$ and function $J_{1}\left(\frac{2 \pi L_{R}}{n_{R}-1}\right)$ only has one zero point, i.e. $P_{3}=\frac{2 \pi L_{R}}{n_{R}-1}=3.8317$. Consequently, $h\left(n_{R}\right)$ has three extreme points in the range of $\frac{\pi}{2}<\frac{2 \pi L_{R}}{n_{R}-1}<2 \pi$, described by $P_{i}, i=1,2,3$.
And then, the second-order derivative of $h\left(n_{R}\right)$ can be computed by

$$
\begin{aligned}
\frac{d^{2} h\left(n_{R}\right)}{d n_{R}{ }^{2}} & =\frac{8 \pi L_{R} J_{0}\left(\frac{2 \pi L_{R}}{n_{R}-1}\right) J_{1}\left(\frac{2 \pi L_{R}}{n_{R}-1}\right)}{\left(n_{R}-1\right)^{3}} \\
& -\frac{8 \pi^{2} L_{R}{ }^{2} J_{1}\left(\frac{2 \pi L_{R}}{n_{R}-1}\right)^{2}}{\left(n_{R}-1\right)^{4}} \\
+ & \frac{4 \pi^{2} L_{R}{ }^{2} J_{0}\left(\frac{2 \pi L_{R}}{n_{R}-1}\right)\left[J_{0}\left(\frac{2 \pi L_{R}}{n_{R}-1}\right)-J_{2}\left(\frac{2 \pi L_{R}}{n_{R}-1}\right)\right]}{\left(n_{R}-1\right)^{4}}
\end{aligned}
$$

Using (27), the second-order derivative in points $P_{i}, i=1,2,3$ is obtained as follow:

$$
\begin{aligned}
& P_{1}:\left.\frac{d^{2} h(n)}{d n_{R}{ }^{2}}\right|_{\frac{2 \pi L_{R}}{n_{R}-1}=2.4048}=-\frac{4.51}{\pi^{2} L_{R}{ }^{2}}<0 \\
& P_{2}:\left.\frac{d^{2} h(n)}{d n_{R}{ }^{2}}\right|_{\frac{2 \pi L_{R}}{n_{R}-1}=5.5201}=-\frac{53.75}{\pi^{2} L_{R}{ }^{2}}<0 \\
& P_{3}:\left.\frac{d^{2} h(n)}{d n_{R}{ }^{2}}\right|_{\frac{2 \pi L_{R}}{n_{R}-1}=3.8317}=\frac{17.48}{\pi^{2} L_{R}{ }^{2}}>0
\end{aligned}
$$

With the discussion above, it is obvious that point $P_{1}$ and $P_{2}$ are local maximum points of $h\left(n_{R}\right)$ and point 
$P_{3}$ is local minimum point. Furthermore, it is obvious from (20) that the extreme points of $h\left(n_{R}\right)$ is same as ones of $g\left(n_{R}\right)$. Consequently, in the range of $L_{R}+1<$ $n_{R}<4 L_{R}+1$, the extreme points of power offset in (25) can be approximately computed by

$$
\widehat{n_{R}}=\left\{\begin{array}{l}
\underset{n_{R} \in \Pi}{\arg \min }\left(L_{\infty}\right), \text { for local minimal point } \\
\underset{n_{R} \in \Pi}{\arg \max }\left(L_{\infty}\right), \text { for local maximum point }
\end{array}\right.
$$

where $\Pi$ is defined as follows:

$$
\left.\Pi=\left\{\left\lfloor\frac{2 \pi L_{R}}{P_{i}}+1\right\rfloor, \mid \frac{2 \pi L_{R}}{P_{i}}+1\right]\right\}, i=1,2,3
$$

where $\lfloor x\rfloor$ and $\lceil x \mid$ denote the maximum integer smaller than $x$ and minimum integer larger than $\mathrm{x}$, respectively. $P_{i}, i=1,2,3$ is the extreme points computed above. (31) can be used to approximately compute the number of receive antenna, in order to achieve the optimal power offset.

\section{Simulation Results}

In the Figure 1 and 2, with the assumption of $\widehat{n_{T}}=2$, power offset is approximately analyzed based on tri-diagonal, five-diagonal, and seven-diagonal toeplitz versus the number of receive antenna, with $L_{R}=5$ in Figure 1 and $L_{R}=6$ in Figure 2. Furthermore, power offset in independent channel is also provided as lower bound for numerical comparison. The approximation trend can be found from Figure 1 and 2. The tri-diagonal toeplitz matrix can be assumably used to characterize the property of power offset over spatially correlated channel. Hence, tri-diagonal toeplitz matrix can be used to approximate the correlation model at the receive node.

The approximation of fitting the curve of tri-diagonal toeplitz matrix determinant is illustrated in Figure 3 and Figure 4 to compare the difference between fitted curves and determinant curves, with $L_{R}=5$ in Figure 3 and $L_{R}=6$ in Figure 4. From Figure 3 and 4, we can find that the fitting curve can exactly approach the extreme points of the determinant of tri-diagonal toeplitz matrix. However, the expression is mathematically tractable.

With the same assumption of $\widehat{n_{T}}=2$, approximation of power offset is shown in Figure 5 and 6, with $L_{R}=5$ in Figure 5 and $L_{R}=6$ in Figure 6. From Figure 5 and 6 , we can find the position of two local minimal points and one local maximum point. With the condition of $L_{R}=5$ and $\widehat{n_{T}}=2$, the local extreme points computed by (31) is $n_{R}=9$ for local maximum point and $n_{R}=7,15$ for local minimal points. With the condition of $L_{R}=6$ and $\widehat{n_{T}}=2$, the local extreme points computed by (31) is $n_{R}=11$ for local maximum point and $n_{R}=8,16$ for local minimal points. The computation results based on (31) coincide with the illustration results in Figure 5 and 6. Hence, all the approximation in this paper is reasonable, including approximation of correlation model and approximation of determinant of tri-diagonal toeplitz matrix.

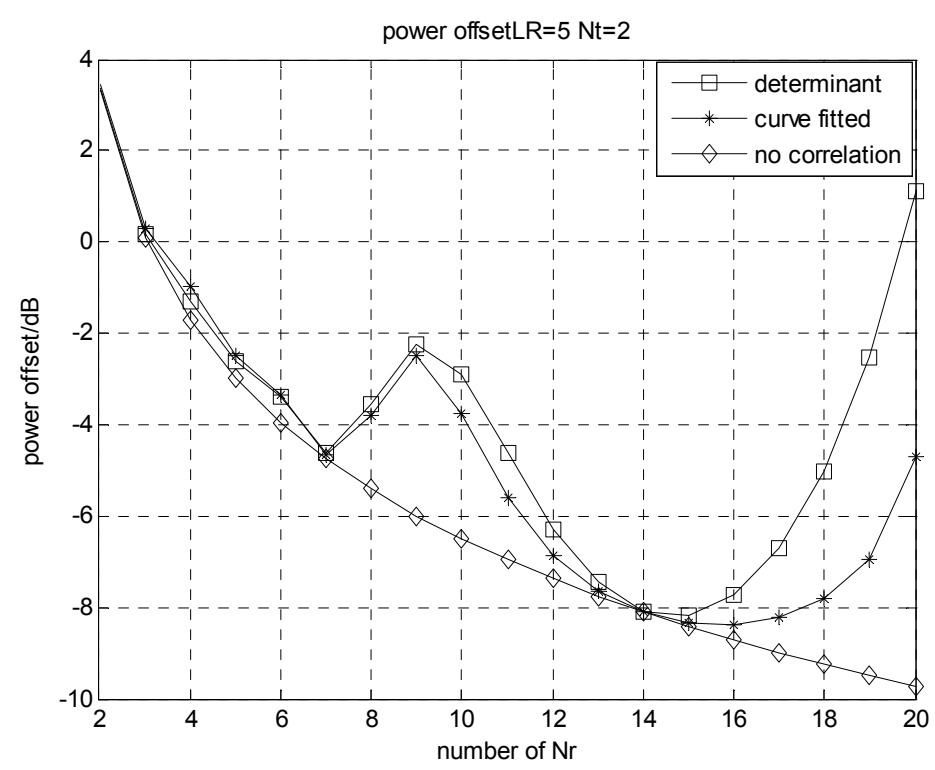

Figure 5. Power offset comparation between accurate and approximate determinant of toeplitz matrix with $L_{R}=5, \widehat{n_{T}}=2$ 


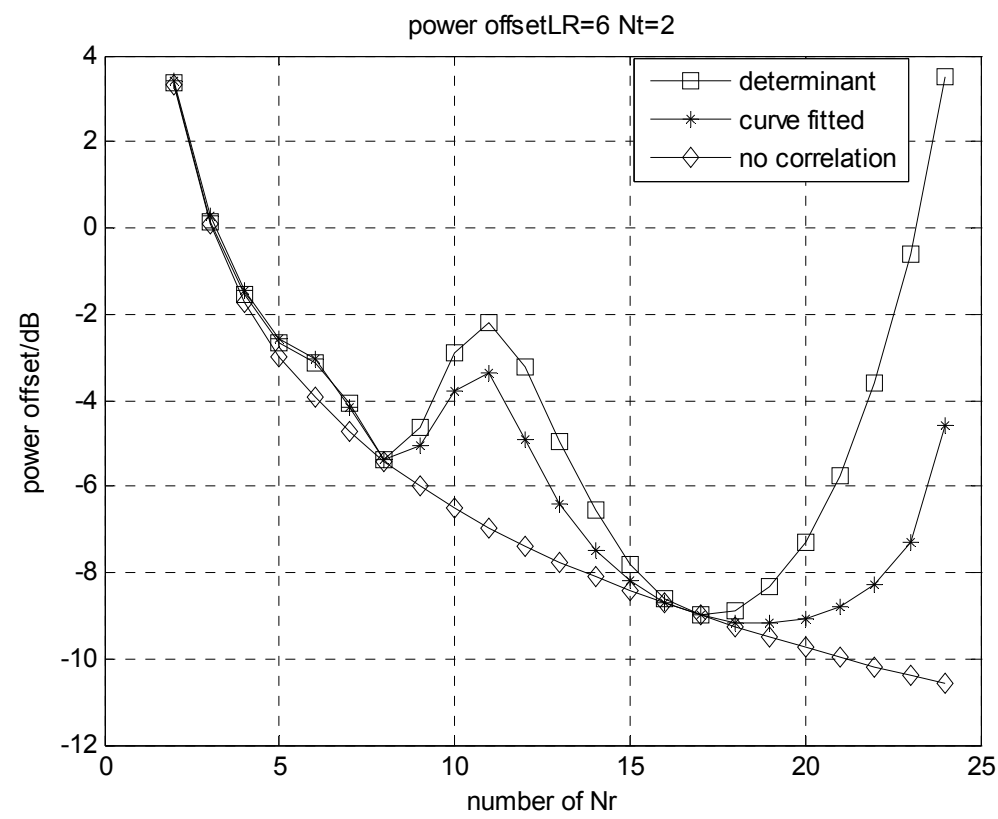

Figure 6. Power offset comparation between accurate and approximate determinant of toeplitz matrix with $\boldsymbol{L}_{\boldsymbol{R}}=6, \widehat{n_{T}}=2$

Similar results are presented in Figure 7 and 8, with the assumption of $\widehat{n_{T}}=4$ and $L_{R}=5$ in Figure 7 while $L_{R}=8$ in Figure 8. With the condition of $L_{R}=5$ and $\widehat{n_{T}}=4$, the local extreme points computed by (31) is $n_{R}=9$ for local maximum point and $n_{R}=7,15$ for local minimal points. With the condition of $L_{R}=8$ and $\widehat{n_{T}}=4$, the local extreme points computed by (31) is $n_{R}=14$ for local maximum point and $n_{R}=10,22$ for local minimal points. The computation results based on (31) coincide with the illustration results in Figure 7 and 8.

Consequently, it is presented in Figure 5 and 7 that the effect caused by the number $\widehat{n_{T}}$ can be neglected. In other words, for small value of $\widehat{n_{T}}$ and in the range of number of receive antenna $L_{R}+1<n_{R}<4 L_{R}+1$, the assumption of $f\left(n_{R}\right) \approx C$ is tenable with neglectable loss of precision. The extreme points of power offset is only determined by $g\left(n_{R}\right)$.

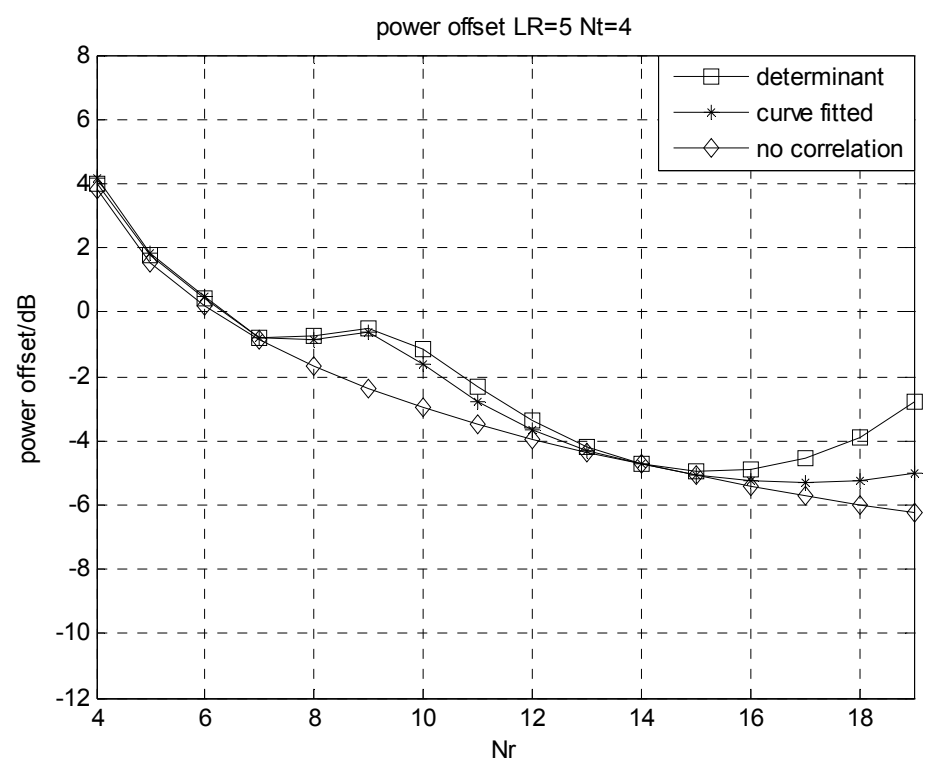

Figure 7. Power offset comparation between accurate and approximate determinant of toeplitz matrix with $L_{R}=5, \widehat{n_{T}}=4$ 


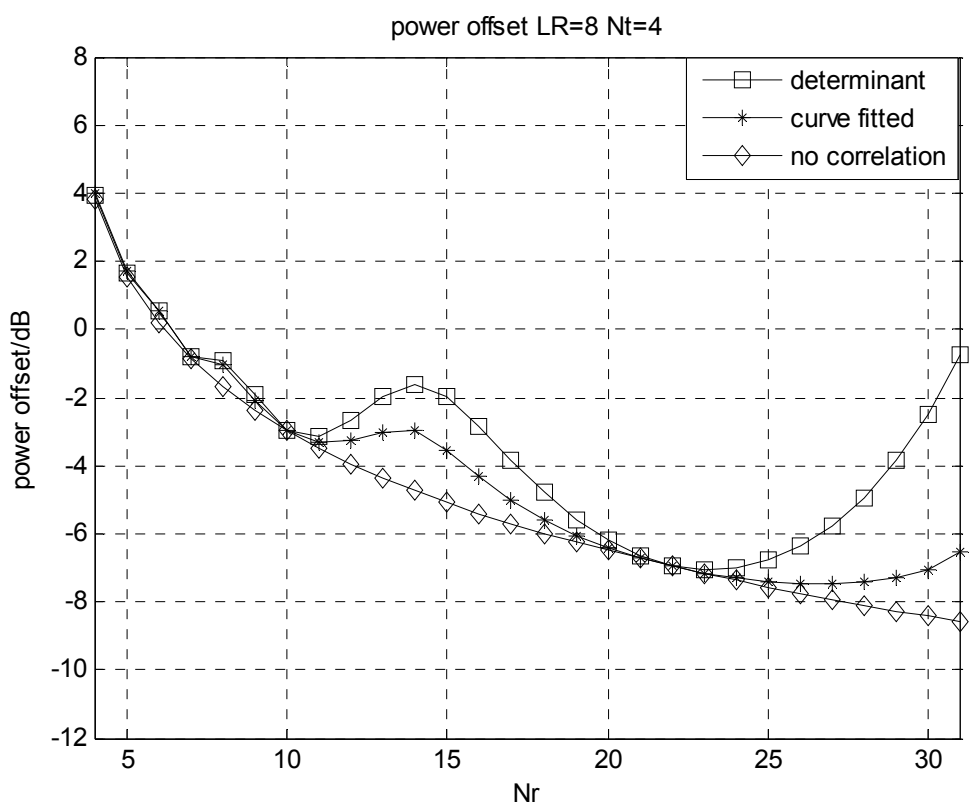

Figure 8. Power offset comparation between accurate and approximate determinant of toeplitz matrix with $\boldsymbol{L}_{\boldsymbol{R}}=8, \widehat{\boldsymbol{n}_{T}}=4$

\section{Conclusions}

Approximate analysis of power offset over spatially correlated channel is proposed to optimize the number of receive antenna with antenna array of fixed length. Antenna correlation matrix is approximated by tri-diagonal toeplitz matrix. The determinant of tri-diagonal toeplitz matrix is reduced to simple style by curve fitting with neglectable loss of precision. Based on the approximation above, the expression of local extreme points of power offset is derived. Simulation results shows that the derived expression is accurate in the local extreme points of power offset, that is to say, all the approximation in this paper is reasonable.

\section{REFERENCES}

[1] TAROKH V, JAFARKHANI H, CALDERBANK A R. Space-time block code from orthogonal designs. IEEE Transactions on Information Theory, Jul. 1999, 45(5): 1456-1467.

[2] BOUBAKER N, LETAIEF K B, MURCH R D. Performance of BLAST over frequency-selective wireless communication channles. IEEE Transactions on Communication, Feb. 2002, 50(2): 196-199.

[3] LOZANO A, PAPADIAS C. Layered space-time receivers for frequency-selective wireless channels. IEEE Transactions on Communication, Jan. 2002, 50(1): 65-73.

[4] BAKIR L, FROLIK J. Diversity gains in two-ray fading channels. IEEE Transactions on Wireless Communications, Feb. 2009, 8(2): 968-977.

[5] POON A, BRODERSEN R, TSE D. N. C. Degrees of freedom in multiple-antenna channels: A signal space approach. IEEE Transactions on Information Theory, Feb. 2006, 51(2): 523-536.

[6] JAFAR S A, SHAMAI S. Degrees of freedom region of the MIMO X channel. IEEE Transactions on Information Theory, Jan. 2008, 54(1): 151-170.

[7] JINDAL N. High SNR analysis of MIMO broadcast channels. ISIT'05, 2005, 2310-2314.

[8] TULINO A M, LOZANO A, VERDU S. High-SNR power offset in multi-antenna Ricean channels. ICC'05, 2005, 683-687.

[9] LOZANO A, TULINO A M, VERDU S. High-SNR power offset in multi-antenna communication. IEEE Transactions on Information Theory, Dec. 2005, 51(12): 4134-4151.

[10] LEE J, JINDAL N. High SNR analysis for MIMO broadcast channels: Dirty paper coding versus linear precoding. IEEE Transactions on Information Theory, Dec. 2007, 53(12): 4787-4792.

[11] ZHENG L Z, TSE D N C. Diversity and multiplexing: A fundamental tradeoff in multiple-antenna channels. IEEE Transactions on Information Theory, May 2003, 49(5): 1073-1096.

[12] TSE D N C, VISWANATH P, ZHENG L Z. Diversity multiplexing tradeoff in multiple-access channels. IEEE Transactions on Information Theory, Sep. 2004, 50(9): 1859-1874.

[13] WAGNER J, LIANG Y C, ZHANG R. On the balance of multiuser diversity and spatial multiplexing gain in random beamforming. IEEE Transactions on Wireless Communication, Jul. 2008, 7(7): 2512-2525.

[14] SHIU D, FOSCHINI G J, GANS M J, ET AL. Fading correlation and its effect on the capacity of multielement antenna system. IEEE Transactions on Communication, Mar. 2000, 48(3): 502-513. 
[15] ABDI A, KAVEH M. A space-time correlation model for multielement antenna systems in mobile fading channels. IEEE J. Select. Areas Communication, Apr. 2002, 20: 550-560.

[16] CHIANI M, WIN M Z, ZANELLA A. On the capacity of spatially correlated MIMO Rayleigh Fading channels. IEEE Transactions on Information Theory, Oct. 2003, 49(10): 2363-2371.

[17] WEI S, GOECKEL D, JANASWAMY R. On the asymptotic capacity of MIMO systems with antenna arrays of fixed length. IEEE Transactions on Wireless Communications, Jul. 2005, 4(4): 1608-1621.
[18] TELATAR I E. Capacity of multi-antenna Gaussian channel. Europe Transactions on Telecommunication, Nov./Dec. 1999, 10: 585-595.

[19] FOSCHINI G J, GANS M J. On the limits of wireless communications in a fading environment when using multiple antenna. Wireless Personal Communication, 1998, 6(3): 315-335.

[20] XU Z, ZHANG K Y, LU Q. Fast algorithm based on toeplitz matrix. Xi'an: Northwest Industrial University, 1999.

[21] ZHUANG Z M, CAI C Q, ET AL. Space-time correlation analysis for MIMO fading channel based on geometric model. Journal of Shantou University, 2008, 23(1): 48-53. 